\title{
Ultra-High Field Magnets for X-Ray and Neutron Scattering Using High Temperature Superconductors
}

\author{
B. L. Winn \\ C. Broholm \\ M.D. Bird \\ B. Breneman \\ M. Coffey \\ R. Cutler \\ R. Duckworth \\ R. Erwin \\ S. Hahn \\ Y. Hernandez \\ K. Herwig \\ L. Holland \\ K. Lonergan \\ Z. Melhem \\ S. Minter \\ C. Nelson \\ P. Paranthaman \\ J. Pierce \\ J. Ruff \\ T. Shen \\ T. Sherline \\ P. Smeibidl
A. Tennant
D. van der Laan
R. Wahle
Y. Zhang

January 2017 


\title{
DOCUMENT AVAILABILITY
}

Reports produced after January 1, 1996, are generally available free via US Department of Energy (DOE) SciTech Connect.

Website http://www.osti.gov/scitech/

Reports produced before January 1, 1996, may be purchased by members of the public from the following source:

\author{
National Technical Information Service \\ 5285 Port Royal Road \\ Springfield, VA 22161 \\ Telephone 703-605-6000 (1-800-553-6847) \\ TDD 703-487-4639 \\ Fax 703-605-6900 \\ E-mailinfo@ntis.gov \\ Website http://classic.ntis.gov/
}

Reports are available to DOE employees, DOE contractors, Energy Technology Data Exchange representatives, and International Nuclear Information System representatives from the following source:

Office of Scientific and Technical Information

PO Box 62

Oak Ridge, TN 37831

Telephone 865-576-8401

Fax 865-576-5728

E-mail reports@osti.gov

Website http://www.osti.gov/contact.html

This report was prepared as an account of work sponsored by an agency of the United States Government. Neither the United States Government nor any agency thereof, nor any of their employees, makes any warranty, express or implied, or assumes any legal liability or responsibility for the accuracy, completeness, or usefulness of any information, apparatus, product, or process disclosed, or represents that its use would not infringe privately owned rights. Reference herein to any specific commercial product, process, or service by trade name, trademark, manufacturer, or otherwise, does not necessarily constitute or imply its endorsement, recommendation, or favoring by the United States Government or any agency thereof. The views and opinions of authors expressed herein do not necessarily state or reflect those of the United States Government or any agency thereof. 
ORNL/TM-2016/712

Workshop on Ultra High Field Magnets for X-Ray and Neutron Scattering

\section{ULTRA-HIGH FIELD MAGNETS FOR X-RAY AND NEUTRON SCATTERING USING HIGH TEMPERATURE SUPERCONDUCTORS}

B. Winn ${ }^{*}$, C. Broholm², M. Bird ${ }^{3}$, B. Breneman ${ }^{4}$, M. Coffey ${ }^{5}$, R. Cutler ${ }^{6}$, R. Duckworth ${ }^{7}$, R. Erwin ${ }^{8}$, S. Hahn ${ }^{9}$, Y. Hernandez ${ }^{8}$, K. Herwig ${ }^{6}$, L. Holland ${ }^{4}$, K. Lonergan ${ }^{10}$, Z. Melhem ${ }^{10}$, S. Minter ${ }^{5}$, C. Nelson ${ }^{11}$, P. Paranthaman ${ }^{12}$, J. Pierce ${ }^{6}$, J. Ruff ${ }^{13}$, T. Shen ${ }^{14}$, T. Sherline ${ }^{6}$, P. Smeibidl ${ }^{15}$, A. Tennant ${ }^{16}$, D. van der Laan $^{17}$, R. Wahle ${ }^{15}$, and Y. Zhang ${ }^{18}$

${ }^{1}$ Oak Ridge National Laboratory, Quantum Condensed Matter Division, Oak Ridge, TN

${ }^{2}$ Johns Hopkins University, Department of Physics and Astronomy and Institute for Quantum Matter, Baltimore, MD

${ }^{3}$ Florida State University, National High Magnetic Field Laboratory, Magnet Science and Technology Division, Tallahassee, FL

${ }^{4}$ General Atomics, San Diego, CA

${ }^{5}$ Cryomagnetics, Oak Ridge, TN

${ }^{6}$ Oak Ridge National Laboratory, Instrument and Source Division, Oak Ridge, TN

${ }^{7}$ Oak Ridge National Laboratory, Fusion \& Materials for Nuclear Systems Division, Oak Ridge, TN

${ }^{8}$ National Institute of Standards and Technology, NIST Center for Neutron Research, Gaithersburg, MD

${ }^{9}$ Florida State University, National High Magnetic Field Laboratory, Applied Superconductivity Center, Tallahassee, FL

${ }^{10}$ Oxford Instruments, Abingdon, UK

${ }^{11}$ Brookhaven National Laboratory, National Synchrotron Light Source II, Upton, NY

${ }^{12}$ Oak Ridge National Laboratory, Chemical Sciences Division, Oak Ridge, TN

${ }^{13}$ CHESS, Cornell University, Ithaca, NY

${ }^{14}$ Lawrence Berkeley National Laboratory, Accelerator Technology and Applied Physics Division, Berkeley, CA

${ }^{15}$ Helmholtz-Zentrum Berlin, High Field Magnet Department, Berlin, DE

${ }^{16}$ Oak Ridge National Laboratory, Neutron Sciences Directorate, Oak Ridge, TN

${ }^{17}$ Advanced Conductor Technologies, LLC, Boulder, CO

${ }^{18}$ SuperPower, Inc., Schenectady, NY

*Correspondence addressed to winnbl@ornl.gov

Date Published: January 2017

Prepared by

OAK RIDGE NATIONAL LABORATORY

Oak Ridge, TN 37831-6283

managed by

UT-BATTELLE, LLC

for the

US DEPARTMENT OF ENERGY

under contract DE-AC05-00OR22725 



\section{CONTENTS}

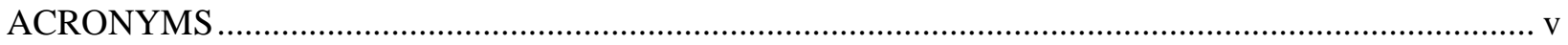

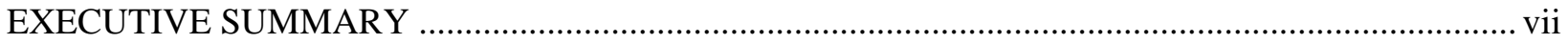

1. THE NEED FOR X-RAY AND NEUTRON SCATTERING AT HIGH MAGNETIC FIELDS ........ 1

1.1 LIMITED SUCCESS BREAKING THROUGH THE 17 T LTS CEILING WITH

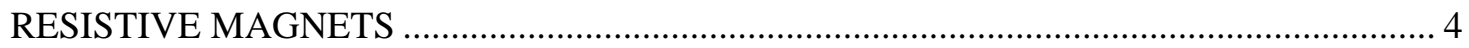

2. HIGH-TEMPERATURE SUPERCONDUCTOR AND MAGNET TECHNOLOGY IS

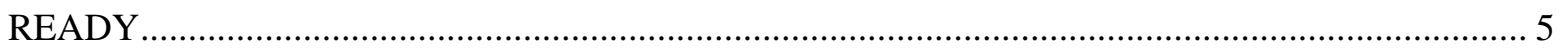

2.1 POTENTIAL FOR UHF HTS MAGNETS AT X-RAY AND NEUTRON USER FACILITIES

3. MAKE UHF HTS MAGNETS AVAILABLE AT X-RAY AND NEUTRON USER

FACILITIES . .8

3.1 PARALLEL MAGNET PROTOTYPE AND TECHNOLOGY DEVELOPMENT ................. 9

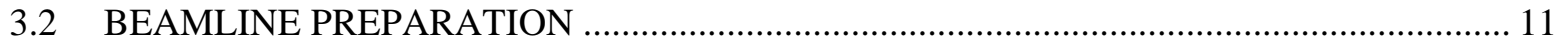

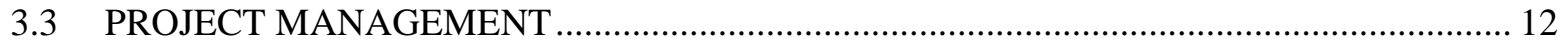

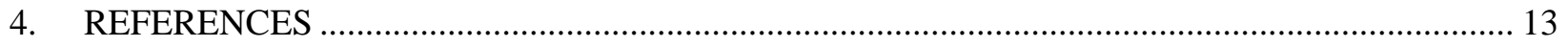

APPENDIX A. CANDIDATE TECHNOLOGIES FOR UHF MAGNETS …..................................... A-1

A.1 INSULATED REBCO TECHNOLOGY FOR NHMFL'S 32 T MAGNET ........................ A-1

A.2 NO-INSULATION REBCO TECHNOLOGY .................................................................. A-1

A.3 MULTI-STRAND HTS CABLE MAGNETS …............................................................... A-2 


\section{ACRONYMS}

ACT

ANL

APS

$\mathrm{B} / \mathrm{T}$

BNL

Bi-2212

Bi-2223

CICC

$\mathrm{CORC}^{\circledR}$

CNCS

CRADA

CD-1

DC

DOE

DOE-BES

DOE-HEP

EXED

HMF

HTS

HYSPEC

HZB

$J_{e}$

LBNL

LTS

MagSci

MIT

MOU

MRI

NDA

NHMFL

NI

NI-ReBCO

NMR

NRC

NSF

ORNL

OST

R2A2

$\mathrm{R}$ \& D

ReBCO

REXS
Advanced Conductor Technologies, LLC

Argonne National Laboratory

Advanced Photon Source

magnetic field/temperature

Brookhaven National Laboratory

$\mathrm{Bi}_{2} \mathrm{Sr}_{2} \mathrm{CaCu}_{2} \mathrm{O}_{8}$

$\mathrm{Bi}_{2} \mathrm{Sr}_{2} \mathrm{Ca}_{2} \mathrm{Cu}_{3} \mathrm{O}_{10}$

cable-in-conduit conductor

conductor on round core

Cold Neutron Chopper Spectrometer

cooperative research and development agreement

Critical Decision 1

direct current

Department of Energy

DOE Basic Energy Sciences Program

DOE Office of High Energy Physics

Extreme Environments Diffraction Beamline

High Magnetic Field Sector for X-Ray Scattering proposal

high-temperature superconductor

Hybrid Spectrometer

Helmholtz-Zentrum Berlin

engineering current density

Lawrence Berkeley National Laboratory

low-temperature superconductor

Committee to Assess the Current Status and Future Direction of High Magnetic

Field Science in the United States

Massachusetts Institute of Technology

memorandum of understanding

magnetic resonance imaging

nondisclosure agreements

National High Magnetic Field Laboratory

no insulation

no-insulation rare earth barium copper oxide

nuclear magnetic resonance

National Research Council

National Science foundation

Oak Ridge National Laboratory

Oxford Superconducting Technology

roles, responsibilities, accountabilities, and authorities

research and development

rare earth barium copper oxide

resonant elastic $\mathrm{x}$-ray scattering 
RIXS

SANS

SESP

SNS

STS

$\mathrm{T}$

UHF

WFO

YBCO restacked rod process

resonant inelastic x-ray scattering

small angle neutron scattering

Superconductivity for Electric Systems Program

Spallation Neutron Source

Second Target Station

tesla

ultra-high fields

work for others

yttrium barium copper oxide 


\section{EXECUTIVE SUMMARY}

$\mathrm{X}$-ray and neutron scattering techniques are capable of acquiring information about the structure and dynamics of quantum matter. However, the high-field magnet systems currently available at X-ray and neutron scattering facilities in the United States are limited to fields of 16 tesla $(T)$ at maximum, which precludes applications that require and/or study ultra-high field states of matter. This gap in capabilityand the need to address it-is a central conclusion of the 2005 National Academy of Sciences report by the Committee on Opportunities in High Magnetic Field Science [1]. To address this gap, we propose a magnet development program that would more than double the field range accessible to scattering experiments. With the development and use of new ultra-high field-magnets, the program would bring into view new worlds of quantum matter with profound impacts on our understanding of advanced electronic materials.

Over the last decade, commercial development of composite conductors based on high-temperature superconductors has led to dramatic progress in high-field superconducting magnets. This progress will culminate in the commissioning of a 32 T user magnet at Florida State University's National High Magnetic Field Laboratory, as well as several revolutionary magnet technologies that are now emerging.

This progress, however, has not yet been applied to the development of steady-state magnets for ultrahigh field-scattering. With suitable development, it appears that scattering experiments in the 25-30 T range are now technically feasible and that even higher ranges will be possible as the conductor technologies mature. However, waiting for the technology to fully mature to the point of commercial deployment would certainly delay critical scientific studies for a decade or more. Instead, active development was strongly recommended in two reports from the National Academy of Sciences in 2005 [1] and 2013 [2].

A planning workshop, Ultra High Field Magnets for X-Ray and Neutron Scattering, was held on August 17-18, 2016 at the Shull Wollan Center at Oak Ridge National Laboratory to gather scientists and engineers so that a plan to act on these recommendations and new opportunities could be prepared. The workshop participants, who also co-authored this paper, include instrument scientists and sampleenvironment experts from neutron and x-ray user facilities at US and German national laboratories, as well as technical experts in the fields of magnets and high-temperature superconducting materials from both US government laboratories and the commercial sector. On the first day of the workshop, speakers and discussions focused on scientific opportunities, magnet specifications, and recent advances in ultrahigh field steady-state magnets. The information was captured in draft sections for this report during the second day. All attendees were later given multiple opportunities to review and recommend revisions to this report.

The workshop participants affirm that the exploration of high-field, stabilized quantum states of matterthrough scattering - is as important as ever. Moreover, technological developments make now the right time to act on a decade of considered recommendations in order to advance the frontier for high-field xray and neutron scattering. To engage the National High Magnetic Field Laboratory, scattering facilities, magnet developers, and high-temperature superconductor composite conductor developers in this endeavor, the workshop participants offer the following specific recommendations.

1. Develop a series of prototype steady-state magnets using high-temperature superconductor composite conductors, with a vertical-bore, split-coil configuration, for user program operation at $\mathrm{x}$-ray and neutron scattering facilities. The first magnet will target $25 \mathrm{~T}$, and each subsequent magnet will achieve a higher maximum field than the previous magnet.

2. Support the development of the prototype magnets with a technology development program. 
3. Prepare x-ray and neutron instruments to accommodate the prototype magnets and realize the scientific progress envisioned in the academy reports.

The result of this program shall be four scientifically productive ultra-high field magnets at both x-ray and neutron scattering facilities. Each prototype magnet produced will leverage and drive technology development in order to achieve higher field than the previous one. In order to financially support this program, we recommend a funding profile of \$8 million per year in 2017 dollars, adjusted for escalation for up to 20 years, plus a one-time investment for important test platforms. 


\section{THE NEED FOR X-RAY AND NEUTRON SCATTERING AT HIGH MAGNETIC FIELDS}

The United States has a broad range of university researchers studying bulk properties of quantum materials in intense magnetic fields, both in the 5-20 T range at university laboratories, and in the 20100 T range at the National High Magnetic Field Laboratory (NHMFL), which hosts around 1,500 users per year. The experiments provide clear evidence of exciting new phenomena when quantum materials are exposed to extremely high magnetic fields. To understand the structure and dynamics of these new phases of quantum materials, neutron and x-ray scattering experiments under high-field conditions are essential. Unfortunately, steady-state magnets available in the United States for neutron and x-ray scattering have maximum fields ranging from 2-16 T, so direct atomic-scale experimental information is presently unavailable for fields beyond $16 \mathrm{~T}$. With the advent of high-temperature superconductor (HTS) magnets, this discrepancy can finally be addressed.

An applied magnetic field affects the outer-shell valence electrons of quantum materials by raising and lowering their spin energies, exerting torques on their orbital and spin magnetic moments, and driving phase transitions. Changes in both structure and dynamics — driven by ultra-high fields (UHF)—reveal critical information about fundamental interactions, which in turn enables a better understanding of collective phenomena in quantum materials. Sample temperatures in the millikelvin (mK) range are typically needed to make full use of the high-field conditions. The ability to change other thermodynamic variables at UHF, such as hydrostatic and uniaxial pressure and impurity doping levels, further expands the accessible multi-dimensional phase space and the range and specificity of possible investigations. Research on quantum materials addresses several of the "grand challenges" [3] that have been identified by DOE committees, particularly:

- “How do we control material processes at the level of electrons?”

- "How do remarkable properties of matter emerge from complex correlations of the atomic or electronic constituents, and how can we control these properties?”

High-field magnets for scattering have been a high priority of two National Academy of Sciences reports. The 2005 report from the "Committee on Opportunities in High Magnetic Field Science” [1] contains the following conclusions and recommendations.

- Conclusion: "US scientists will be unable to access a wealth of science opportunities if high magnetic field instrumentation is not provided at the Spallation Neutron Source and at the nation's third-generation light sources.”

- Recommendation: "New instruments for studying the neutron and x-ray scattering properties of materials in high magnetic fields should be developed in the United States."

- Recommendation: "A consortium should be established to foster the development of magnet technology.”

The 2013 report on "High Magnetic Field Science and Its Application in the United States: Current Status and Future Directions” (MagSci) [2] contains the following recommendation:

- Recommendation: "New types of magnets should be developed and implemented that will enable the broadest possible range of x-ray and neutron scattering measurements in fields in excess of $30 \mathrm{~T}$. This requires, as a first step, the expeditious procurement of modern 10-16 T magnet/cryostat systems for US facilities, together with the recruitment of low-temperature/high-field specialists. 
Second, a $40 \mathrm{~T}$ pulsed-field magnet should be developed with a repetition rate of $30 \mathrm{~s}$ or less. Third, building on the development of a high-temperature all-superconducting magnet, which was recommended earlier, a wider-bore 40 T superconducting DC magnet should be developed specifically for use in conjunction with neutron scattering facilities. New partnerships among federal agencies, including the Department of Energy, the National Institute of Standards and Technology, and the National Science Foundation, will likely be required to fund and build these magnets, as well as to provide the funds and expertise that will be needed to operate these facilities for users once they are built.”

As such, this white paper serves as a detailed proposal to act on the recommendations from the academy reports and to deliver the capability to conduct scattering experiments at ultra-high magnetic fields.

The x-ray and neutron scattering communities currently conduct experiments using commerciallyavailable low-temperature superconductor (LTS) magnets. The first commercial split-pair magnet was manufactured by Oxford Instruments in 1963 and delivered to Dr. Butt of Birkbeck College, London University [4]. In 1985, Cryomagnetics delivered a neutron scattering magnet to what is now the Aalto University Low Temperature Laboratory in Helsinki, Finland. Unlike magnets used for magnetic resonance imaging (MRI) or nuclear magnetic resonance (NMR) - applications where tens of thousands of units have been installed - the scattering magnet market is quite small with only a few dozen systems worldwide.

Magnets at x-ray and neutron scattering facilities have unusual and challenging requirements. These magnets and their cryostats must allow for a wide variety of incident and scattered beam geometries, including the split-coil geometry, with large forces across the gap and high fields at coil ends. Neutrons and different energy ranges of $\mathrm{x}$-rays require specialized optics, including windows, collimators, and absorbers. The sample often needs to be positioned and oriented while in the magnet, and extremes of temperature and pressure may also be necessary. To enable high throughput and efficient use of scattering facilities, expedient sample transfer and reestablishment of field and other sample conditions is necessary. Different experimental techniques require different field orientation with respect to the incident beam. Some x-ray experiments must account for sample heating and damage by $\mathrm{x}$-rays. Some neutron experiments need to preserve neutron polarization and therefore must avoid passing the polarized beam through zero-field regions. These factors impact magnet design and were identified as significant difficulties in the 2013 MagSci report [2]. Therefore, each magnet is developed specifically to meet the requirements of the facility placing the order. Delivery can take 1-3 years. Due to the small market, the complicated geometry, and the technical requirements, very few companies can deliver these specialty magnets.

Scattering studies using LTS magnets with fields up to $17 \mathrm{~T}$ have generated significant amounts of highimpact science. Neutron scattering using LTS magnets played a key role in understanding magnetic monopoles in spin ice [5], vortex matter in high-temperature and heavy fermion superconductors [6], [7], and the Luttinger liquid of quantum spin chains [8], [9], [10], [11]. Neutron scattering using LTS magnets has also been employed to determine complex magnetic interactions in quantum magnets and thereby constrain theoretical analysis [12], [13]. X-ray scattering techniques, including single-crystal and powder diffraction, resonant scattering, and high-energy scattering, have been used to study a variety of systems using commercially available LTS magnets. Scientific topics include the spin-Peierls transition [14], magneto-calorics [15], magneto-electrics [16], magnetic order in rare earth intermetallics [17], [18], and competing order in cuprates [19].

We recommend that the proposed program concentrate on developing magnets with the vertical-field, split-coil geometry. Two basic solenoidal magnet geometries are used for x-ray and neutron scattering to maximize the field and to position the coils out of incident and scattered beam paths: (1) the vertical-field, 
split-coil geometry and (2) the horizontal-field, conical-bore geometry that may optionally split the coil to accommodate top-loading sample environments. Although some scattering experiments require the field direction to be parallel or near parallel to the incident beam-especially some reflectometry experiments and some small angle scattering experiments - and other scattering experiments require the field direction to be oriented near the momentum transfer vector, which is usually in the horizontal plane, most commercial LTS magnets purchased—and most scattering experiments performed —employ the verticalfield split-coil geometry. Significant experience with top-loading, sample-environment systemsincluding ${ }^{3} \mathrm{He}$ and dilution refrigeration inserts, pressure cells, and high-temperature inserts - compatible with the vertical-field, split-coil configuration has made multi-extreme thermodynamic conditions practical at user facilities. Stray fields at the side of a vertical-field split-coil magnet are generally lower field than those near the axis of a horizontal-field magnet and are easier to mitigate with shielding or compensation coils; the impact of these stray fields are expected to increase as magnets achieve higher field-at-sample. Symmetrically-placed, optical-access windows in a vertical-bore, split-pair magnet offer the additional opportunity to implement complementary techniques (e.g., resonant elastic x-ray scattering [REXS] and resonant inelastic x-ray scattering [RIXS]), or potential pump-probe (e.g. optical, Terahertz) experiments on the same instrument.

For single crystal diffraction and inelastic scattering experiments, the vertical-bore, split-coil configuration provides additional advantages. Access to reciprocal space requires rotating the single crystal around an axis that is perpendicular to the scattering vector. To maintain thermodynamic conditions, the sample rotation axis must coincide with the field axis. Both solenoidal geometries can satisfy these conditions, but they access different regions of reciprocal space at different resolutions as a function of scattering angle ranges and neutron or $\mathrm{x}$-ray energy range. Access to more of the $\pm 180^{\circ}$ scatter range in the plane that is perpendicular to the rotation axis-compared to only the forward and back scattering afforded by the horizontal conical-bore geometry-enables more flexibility to simultaneously optimize momentum transfer range and resolution. Also, for a fixed incident energy, a more comprehensive region of reciprocal space in the horizontal scattering plane can be measured. In addition, the relative ease of rotating a top-loading system with respect to a vertical field magnet, while preserving the sample orientation with respect to field, allows sweeping through the observable scattering plane in reciprocal space, which is indispensable for single crystal work. For x-rays, the ability to change incident photon polarization enables analysis of order-parameter symmetry without the need for rotation around the momentum transfer vector; however, this does require the applied field to remain fixed along a given crystallographic axis during polarization-dependent $\mathrm{x}$-ray scattering studies, as afforded by the verticalfield, split-coil configuration. A selection of advantages and disadvantages for each geometry is presented in Table 1.

Table 1. Overview of field configurations for similar high-field scattering experiments. Green text indicates a benefit, and red text indicates a challenge.

\begin{tabular}{llll}
\hline \multicolumn{1}{c}{ Configuration } & Scattering geometry & Sample environment & \multicolumn{1}{c}{ Magnetic field } \\
\hline $\begin{array}{l}\text { 1. Vertical split coil: } \\
\text { field perpendicular to } \\
\text { scattering plane }\end{array}$ & $\begin{array}{l}\text { Large range of } 2 \theta \text { for } \\
\text { diffraction and inelastic } \\
\text { scattering }\end{array}$ & $\begin{array}{l}\text { Enters along vertical } \\
\text { field axis }\end{array}$ & $\begin{array}{l}\text { Large forces across } \\
\text { split and large fields } \\
\text { near split }\end{array}$ \\
$\begin{array}{l}\text { 2. Horizontal conical } \\
\text { solenoid with field } \\
\text { perpendicular to Q }\end{array}$ & $\begin{array}{l}\text { Limited range of } \\
\text { scattering angles. } \\
\text { Useful for some small } \\
\text { angle scattering and } \\
\text { reflectometry }\end{array}$ & $\begin{array}{l}\text { Enters near horizontal } \\
\text { axis }\end{array}$ & $\begin{array}{l}\text { Limited field reduction } \\
\text { relative to solenoid. } \\
\text { Larger and more } \\
\text { complex stray fields in } \\
\text { the scattering plane }\end{array}$ \\
\hline
\end{tabular}


Because of these advantages, higher demand for the vertical-field, split-coil geometry is expected to persist as magnets with higher fields are developed. However, to achieve higher fields with a split-coil configuration, significantly higher forces in the coil gaps will present significant technical challenges. An emphasis on a vertical-bore, split-coil magnet systems will push important developments of the mechanical support within the split-coil gap for even higher-field scattering magnets.

\subsection{LIMITED SUCCESS BREAKING THROUGH THE 17 T LTS CEILING WITH RESISTIVE MAGNETS}

While LTS materials like $\mathrm{Nb}_{3} \mathrm{Sn}$ and NbTi are suitable for the production of high-field magnets in the 517 T range [20], they are limited by their upper critical fields. Future x-ray and neutron scattering experiments require fields above $20 \mathrm{~T}$ and will eventually require fields exceeding $30 \mathrm{~T}$. Therefore, either HTS or resistive materials are required for steady-state operation above $17 \mathrm{~T}$.

While there has been no progress towards enabling scattering experiments that employ higher-field magnets at steady-state in the United States, access to neutron scattering at $26 \mathrm{~T}$ was recently achieved at Helmholtz-Zentrum Berlin (HZB) in Germany [21], with the magnet provided by the NHMFL, a US laboratory. The collaborative effort to develop this 26 T system began in earnest in 2007, with the goal to achieve the highest steady-state field to date for any neutron scattering facility using a single magnet. This magnet is now the centerpiece of the Extreme Environments Diffraction Beamline (EXED), an instrument that can be reconfigured for a variety of techniques [22]. The 26 T magnet at HZB employs a resistive/superconducting hybrid design, with an "outsert" that uses LTS cable-in-conduit conductor (CICC) combined with a 4 MW water-cooled Florida-Bitter insert, run in series with a current of up to 20 kA. The magnet's support systems (e.g., power supplies, chilled water system, cryogenic system, controls) are housed in a dedicated four-story infrastructure building adjacent to the guide hall. The magnet could be upgraded to $30 \mathrm{~T}$ at $20 \mathrm{kA}$ by upgrading the power and cooling plant to $8 \mathrm{MW}$ and replacing the relatively inexpensive resistive coils. Since the inner coil is a water-cooled resistive system, the natural choice for bore temperature was warm. To achieve the highest field and to most easily accommodate the hybrid configuration, a horizontal bore with converging-diverging conical access and no split was chosen (see configuration 2 in Table 1). New customized sample environment equipment was designed and commissioned, with access to the magnet from the upstream end, to enable temperatures down to $500 \mathrm{mK}$, or to $1.8 \mathrm{~K}$ with vertical axis rotation. Development has begun to reach approximately $50 \mathrm{mK}$ with a dilution refrigeration insert. To date, techniques employed at EXED include single crystal neutron diffraction and small angle neutron scattering (SANS), and the recent installation of a new detector vessel and imminent delivery and commissioning of a chopper will enable direct geometry spectroscopy as well [23]. The scientific impact of experiments recently performed at EXED is expected to be high, as user teams prepare publications describing exciting new results. Unfortunately, the research reactor at HZB will shut down in 2019, once again leaving the neutron scattering community bereft of any access to steady-state magnetic fields greater than $17 \mathrm{~T}$.

In 2006, the National Science Foundation (NSF) funded a design study of a similar magnet and instrument concept, called ZEEMANS, for Oak Ridge National Laboratory's (ORNL) Spallation Neutron Source (SNS). The co-principal investigators were at Johns Hopkins University, the SNS, and the Massachusetts Institute of Technology (MIT). There was a major sub-award to the NHMFL where much of the work was conducted. Although a hybrid strategy was clearly the best choice 10 years ago, there has been tremendous improvement of commercial HTS conductors in the past decade, as well as dramatic progress in the technology of magnets based on these conductors. The resulting magnet should attain at least a $25 \mathrm{~T}$ field while consuming orders of magnitude less power and requiring dramatically less space than a hybrid system. Finally, while the capital cost of the first HTS version might be comparable to what 
HZB paid for their magnet and infrastructure, subsequent copies would not require the R \& D activities and contingencies would presumably be lower. As a result, a copy could be built at significantly lower cost.

The HZB/EXED project serves as a model of a how to obtain the coil system for a high-field magnet outside of normal commercial channels. The project management was exemplary, but a commitment to established technologies was made in order to minimize project risks. This white paper proposes a different approach that addresses the risk of developing magnets head on by refining key technologies and developing a series of prototype magnets in parallel within an integrated R \& D program.

Pulsed magnets that exceed $26 \mathrm{~T}$ were recently introduced to both $\mathrm{x}$-ray and neutron scattering communities. Pulsed-magnet X-ray scattering experiments reaching peak fields of 30-40 T have provided a glimpse of intriguing phenomena, including the symmetry-breaking role of nematic order in pnictides [24], the magneto-elastic behavior of a spin liquid [25], Jahn-Teller and valence transitions [26], [27], phase separation [28], and a complex phenomenology of charge-density waves and superconductivity in an HTS [29]. Pulsed magnet neutron Laue diffraction has been employed to study multiferroics [30] and a field-induced antiferromagnetic state in the enigmatic heavy fermion system $\mathrm{URu}_{2} \mathrm{Si}_{2}$ [31].

While these investigations underscore the scientific promise and urgency of scattering studies in a high magnetic field, pulsed magnets are only compatible with elastic scattering and only when enough x-rays or neutrons are scattered during the brief time that the sample is at field (approximately 5 ms pulse width). The pulsed magnets in use at $\mathrm{x}$-ray and neutron scattering facilities that have proven serviceable to date (Nojiri's coils operated at several facilities and the Laboratoire National des Champs Magnétiques Intenses system [32] at the Institut Laue-Langevin) can only use a tiny fraction of the x-ray or neutron beam $(<0.005 \%)$ because most of the time is spent waiting for the magnet to cool down after the previous pulse (around 6-8 minutes between coil pulses). These experiments are also challenging due to the limited fatigue life of the highly-stressed magnet coils (around 5000 shots) and the deleterious effects on the sample and the experiment caused by eddy currents, vibrations, and hysteresis. Therefore, steady-state magnets are essential for most experiments, especially for inelastic scattering experiments.

\section{HIGH-TEMPERATURE SUPERCONDUCTOR AND MAGNET TECHNOLOGY IS READY}

The first HTS material was discovered 30 years ago (1986). The path from discovery to viable UHF conductor and then UHF magnet technology has been long and circuitous with contributions from major governmental and commercial players in the United States, Japan, Germany, and elsewhere. For around 15 years the largest US effort was the US Department of Energy's (DOE) Superconductivity for Electric Systems Program (SESP) [33]. SESP originally started in the mid-1990s, with annual funding ranging between \$25 million and \$40 million per year, and ended in 2010 after being sponsored by three different DOE offices. Other US government efforts were funded by the DOE Office of High Energy Physics (DOE-HEP) and the NSF. The SESP focused on the high-temperature aspects of the HTS materials, pursuing utility-relevant devices like power transmission cables, transformers, and generators that would improve grid stability, efficiencies, and energy densities in urban areas with limited space and growing power demands [34]. DOE-HEP and NSF focused more on the high-field aspects of the HTS materialspursuing UHF magnets at lower, liquid helium temperatures.

Fortunately, what emerged from this effort was a collection of HTS conductors that now seem ready for UHF magnets, including user magnets like NHMFL's new $32 \mathrm{~T}$ magnet and exciting new prototype magnets with brand new technology. While many HTS materials exist, only rare earth barium copper oxide ( $\mathrm{ReBCO}$ ), $\mathrm{Bi}_{2} \mathrm{Sr}_{2} \mathrm{Ca}_{2} \mathrm{Cu}_{3} \mathrm{O}_{10}(\mathrm{Bi}-2223)$, and $\mathrm{Bi}_{2} \mathrm{Sr}_{2} \mathrm{CaCu}_{2} \mathrm{O}_{8}(\mathrm{Bi}-2212)$ currently show the potential 
of carrying high engineering current density $J_{e}$ — which accounts for the cross sectional area of the entire composite conductor - in the extreme conditions associated with UHF coils.

A new version of ReBCO tape developed in 2007 by SuperPower, Inc. (a US corporation) consisted of an yttrium barium copper oxide (YBCO) film (approximately 1 micron) on a Hastelloy substrate (approximately 50 microns) along with buffer layers and copper stabilizer (approximately 40 microns). The resulting tape provided an unprecedented combination of strength ( $>600 \mathrm{MPa}), J_{e}\left(3000 \mathrm{~A} / \mathrm{mm}^{2}\right.$ at 20 $\mathrm{T}$ ), flexibility (11 $\mathrm{mm}$ bend-diameter without degradation), protection, and solderability (approximately 40 microns of copper plating) in a conductor that is fully reacted in the factory so that post-winding treatment is no longer required. Most of the previously developed 20-T conductors could not meet any of these performance metrics, much less all of them. SuperPower then built a small coil that was tested to 7 T inside a $20 \mathrm{~T}, 20 \mathrm{~cm}$ resistive magnet at the NHMFL, thereby surpassing the NHMFL's $5 \mathrm{~T}+20 \mathrm{~T}$ " $\Delta \mathrm{B}$ " record that used a much more fragile conductor [35], [36]. These results took the community by storm, and so the NHMFL, some of the magnet groups funded by DOE-HEP, and various foreign laboratories also started working on magnets based on reinforced ReBCO tape. Worldwide, several other companies started fabricating ReBCO tape to compete with SuperPower.

Sumitomo had been making Bi-2223 tape for many years and were, annually, manufacturing the most HTS conductor of any supplier when this new high-strength ReBCO tape became available. They saw the enthusiasm it was generating and contracted with a US company (Solid Materials Solutions) to reinforce their Bi-2223 to make it more competitive with the SuperPower ReBCO. Sumitomo's reinforced Bi-2223 tape became available in 2013 [37]. Various groups worldwide, including NHMFL, are now also working on developing magnet technology based on reinforced Bi-2223 tape.

In 2003, a Bi-2212 multifilamentary round wire was developed by Oxford Superconducting Technology (OST) in New Jersey. In 2011, the NHMFL discovered that voids in Bi-2212 were the major current limiting mechanism [38]. This work led to a 100-bar overpressure heat treatment-similar to the process used by Sumitomo to make Bi-2223 tapes - that increased the $J_{e}$ of OST's Bi-2212 conductor by around 3 times to $>720 \mathrm{~A} / \mathrm{mm}^{2}$ at $4.2 \mathrm{~K}$ and $20 \mathrm{~T}$ after a $\$ 2$ million investment from DOE-HEP into a Very High Field Magnet Collaboration [39]. This material has much better electromagnetic and mechanical isotropy than ReBCO and allows fabrication of Rutherford cables. The multifilamentary nature promises better spatial homogeneity for NMR magnets than magnets using ReBCO. With the support of the DOE-HEP conductor development program (now the US HEP Magnet Development Program), OST further improved the $J_{e}$ of this conductor by $30 \%$ in 2016 and now demonstrates long-length production up to $2.5 \mathrm{~km}$. However, this conductor is mechanically weak, relative to ReBCO tapes, and requires carefully controlled high-temperature reactions for coil fabrication. Therefore, any coil technology will need to address these issues, and for the moment ReBCO tape seems to be the preferred path to UHF magnets for scattering. The NHMFL contracted with Solids Materials Solutions in 2016 to reinforce the Bi-2212 round wire in order to provide a high strength version suitable for UHF magnets.

Several technologies - that employ some of these already-existing HTS composite conductors-are candidates for the development of the series of prototype steady-state magnets which are proposed for this program. The first and most established technology is that used for the NHMFL 32 T magnet, which employs an HTS insert wound from an insulated ReBCO tape and an LTS outsert [40]. The second, and as-of-now the most promising, technology employs no-insulation ReBCO (NI-ReBCO) coils [41]. There are several other potential technologies including single-strand $\mathrm{Bi} 2223$ [42] and Bi2212 [39], and multistrand HTS cables made of ReBCO [43] or Bi2212 [44]. More details about these candidate technologies may be found in Appendix A. 


\subsection{POTENTIAL FOR UHF HTS MAGNETS AT X-RAY AND NEUTRON USER FACILITIES}

While numerous UHF HTS test coils have been built over the past 30 years, it is only in the past few years that real user magnets are being developed. In the summer of 2016, NHMFL completed construction of a solenoid based on ReBCO tape that is intended to provide $32 \mathrm{~T}$ for condensed matter physics experiments. Also, the Tohoku Magnet Lab recently completed a $24.5 \mathrm{~T}$ magnet based on Bi2223. Other general-purpose solenoids are expected to follow shortly. Some HTS magnets have been produced commercially, but only for moderate field strengths of up to around $8 \mathrm{~T}$ [45]. These magnets typically take advantage of the higher operating temperature threshold of the HTS materials—as compared to the lower temperature threshold of LTS materials - and are sometimes cooled with liquid nitrogen. Work on UHF magnets is still conducted primarily at government laboratories - with the exception of Bruker, which is developing $28 \mathrm{~T}(1.2 \mathrm{GHz})$ NMR magnets based on ReBCO with significant German government subsidies.

While HTS magnets will require significantly less support infrastructure and electric power compared to the hybrid magnet at HZB, they are expected to require more support systems than today's LTS magnets. A magnet that closely emulates the NHMFL 32 T HTS/LTS magnet will need a pair of racks to support the batteries required to energize the quench protection system on the HTS coils. The HTS magnet will also consume more liquid helium per day, requiring either daily manual refills or, preferably, a separate dewar with 5-8 closed-cycle refrigerators with compressors to support a recondensing system and autorefill capability. These support systems take up space and have additional power requirements and, as a result, may be difficult to use in parallel with other required support systems (e.g., dilution refrigeration inserts). To account for stray fields emanating from new higher-field magnets, existing instruments may need modification or new instruments may need to be built. Stray field from higher-field magnets may be higher than that of lower-field solenoid magnets; however, if the coils can be made more compact because of higher $J_{e}$, or if active shield coils are included in the HTS magnet design, then the stray field can be reduced.

A preliminary design study of 21-25 T magnets in the split-coil configuration, using the same insulated ReBCO technology as the $32 \mathrm{~T}$ magnet, has been performed at the NHMFL under a contract from HZB. A modified version better suited for $x$-rays has also been developed by the NHMFL working with the Advanced Photon Source (APS). Preliminary designs of NI-ReBCO magnets have also been developed.

In 2016, with the $32 \mathrm{~T}$ magnet nearing final assembly and significant progress made with NI coil technology, several people from the NHMFL and APS/Argonne National Laboratory (ANL) prepared a joint proposal with a team of external researchers for a High Magnetic Field Sector for X-Ray Scattering (HMF) project, which would be one of the world's leading beamlines to be developed as part of the APSU upgrade project [46]. This beamline would be based around a one-of-a-kind, split-pair, all-HTS, 25-T magnet for hard x-ray scattering experiments. Of the 36 whitepapers submitted, 14 were selected in March 2016 by APS-U project coordinators to become full proposals. The HMF whitepaper was one of these 14 . A team of nine investigators with expertise in condensed matter physics, $\mathrm{x}$-ray science, and high-field magnet design prepared a full proposal with contributions from dozens of additional stakeholders from the community at large. This proposal reviewed very favorably on scientific merit, but the costs and risks involved in the design of the magnet were deemed too high to be within the scope of the APS-U project. In addition, there was some concern that the beamline did not fully exploit the unique source characteristics of the APS-U [47]. APS management remains committed to the idea of the HMF project, and encouraged the proposal team to seek additional sources of funding to develop the magnet.

In 2015-2016, ZEEMANS was incorporated into the Second Target Station (STS) project at ORNL's SNS. The new neutron source characteristics will better complement the variety of scattering techniques planned for ZEEMANS. Although the earlier design concept called for a horizontal-bore, no-split 
configuration, the primary magnet is now envisioned as a vertical-bore, split-coil system. For UHF SANS experiments, a separate horizontal conical bore magnet may be built and used at ZEEMANS in the future. At this point, with the 32-T magnet construction completed and the lessons learned from the recent NHMFL design study for HZB, it appears HTS technology is the route forward. Currently, both the magnet and the instrument are considered critical parts of the STS project, and the intent is to fund both through DOE Basic Energy Sciences Program (DOE-BES) via the STS capital project. The STS project has not yet submitted a proposal for critical decision 1 (CD-1).

\section{MAKE UHF HTS MAGNETS AVAILABLE AT X-RAY AND NEUTRON USER FACILITIES}

In order to provide UHF magnets at neutron and x-ray scattering facilities as soon as possible, we must actively refine and mature the most promising technologies and embrace the costs and risks associated with sustained research and development. The effectiveness of the proposed magnets for routine operation and flexible sample environment configurations, as part of a user program, hinges on this development program being able to operate as a partnership among the magnet development laboratory, x-ray and neutron scattering communities, user magnet manufacturers, and HTS manufacturers. Therefore, we propose a plan with the following components, as laid out in the Gantt chart in Figure 1:

1. Develop a series of prototype steady-state magnets using HTS, with a vertical-bore split-coil configuration, for user program operation at x-ray and neutron scattering facilities. The first magnet will target $25 \mathrm{~T}$, and each subsequent magnet will achieve a higher maximum field than the previous magnet.

2. Support the prototype magnets with a technology development program.

3. Prepare x-ray and neutron instruments to accommodate the prototype magnets and realize the scientific progress envisioned in the academy reports.

Due to the significant technical uncertainties, what we propose can in no way be compared to traditional LTS magnet procurements. These prototype magnets, after initial testing at the magnet development laboratory, are intended to be shipped to user facilities for ultimate use in their user programs. The prototype magnets proposed are merely the harbingers of other UHF magnet systems we hope to see at all $\mathrm{x}$-ray and neutron scattering facilities in the next 20 years. Although there might be some desire to wait until one can procure such magnets from a commercial vendor in a fixed-price, guaranteed performance situation, that would only happen if we wait until someone else (in Europe or Asia) assumes the risk and reaps the scientific and technological benefits associated with developing and producing the first wave of these magnets.

We anticipate a need for continuous investment over the next 15 years, with \$2 million per year for magnet component prototyping and testing, and another \$2 million per year for associated conductor refinement and conductor manufacturing improvements, with adjustments for inflation as part of the technology development program (item 4 of the Gantt chart in Figure 1). We also propose investing $\$ 4$ million per year after a brief initial evaluation of magnet technologies, in order to manufacture a series of prototype user magnets which will be accommodated on x-ray and neutron instruments to drive a world-leading scientific program based on scattering experiments at ultra-high magnetic fields. Both the technology development program and the magnet prototyping programs should be funded by R \& D contracts. In addition, initial capital investments in important test platforms are required. We do not propose that this program fund or manage x-ray or neutron instrument upgrades or new instruments directly; user facilities will be responsible for allocating funds and managing separate upgrades or new capital projects through facility-specific processes outside the scope of this program. What this program 
will provide is a separate funding and management system for the prototype magnets, bringing instrument capital costs closer to average levels. Should this path become established, it will provide a mechanism for mitigating the risk inherent in building beamlines at x-ray and neutron scattering facilities for otherwise unproven magnets.

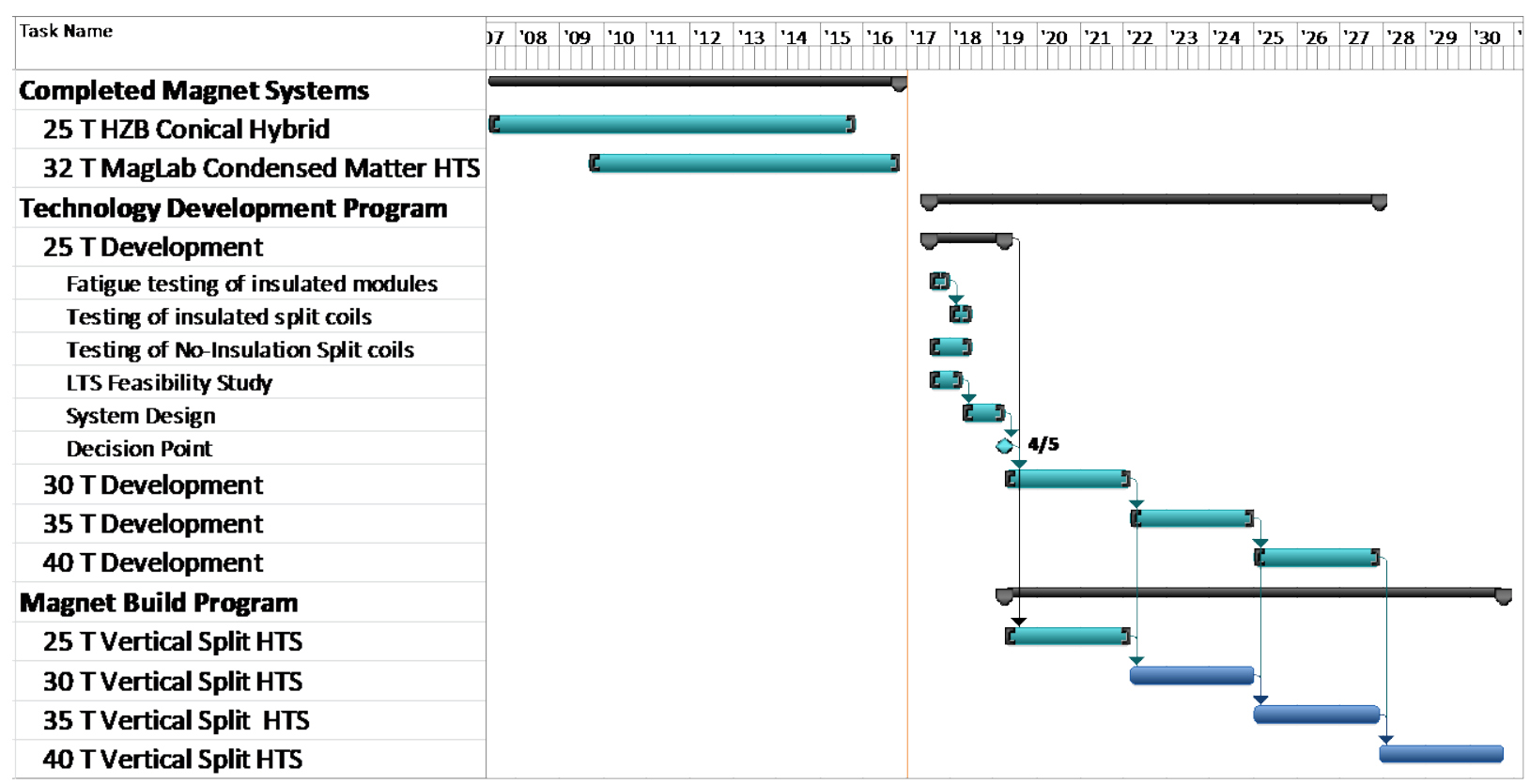

Figure 1. Gantt chart for development of UHF scattering magnets for US DOE-BES laboratories.

\subsection{PARALLEL MAGNET PROTOTYPE AND TECHNOLOGY DEVELOPMENT}

The first magnet is intended to be a $25 \mathrm{~T}$ vertical-field split system. We already have a head start thanks to the NHMFL's $32 \mathrm{~T}$ magnet and design studies. We are currently considering two competing technologies for the first magnet prototype: (1) insulated ReBCO and (2) NI-ReBCO. In order to decide between these technologies as rapidly as possible prior to starting construction for the first magnet prototype, six development tasks_-listed below—-need to be completed and a decision date agreed upon.

1. Perform fatigue testing of the "prototype" coils from the $32 \mathrm{~T}$ magnet project. While a great deal of fatigue testing of tape, joints, and other components was conducted as part of the $32 \mathrm{~T}$ project, there was not time to fatigue test actual modules.

2. Build a split test ReBCO coil and fatigue cycle it. As part of the $32 \mathrm{~T}$ project, several small solenoids were tested, but no split pairs of coils were tested. We need to demonstrate reliable terminals on the gap side of the coils and show that the slip surfaces between the coils and the mid-plane spacer are reliable prior to committing to the full-scale user magnet and beamline.

3. Build and test split coils using NI-ReBCO technology. Several NI-ReBCO coils have been tested at the NHMFL, but there was limited quench testing and no fatigue testing; also, no NI split pairs were available for testing. This technology should be demonstrated on a small and mid-sized scale (4 T, $245 \mathrm{~mm}-26 \mathrm{~T}, 35 \mathrm{~mm}$ ) prior to committing to the full-scale user magnet and beamline. 
4. Conduct design studies of the LTS sections of the $25 \mathrm{~T}$ user magnet. The magnet will likely include an LTS section which we anticipate having developed in the commercial sector. While $\mathrm{Nb}_{3} \mathrm{Sn}$ and NbTi coil technology is far more advanced than HTS coil technology, to our knowledge no commercial supplier has delivered a split LTS system similar to what is needed here. We should commission design studies from the commercial sector to determine the design and costs.

5. Exactly two years after the start of this program, make decisions based on any results of items 1-4 concerning: whether the 25 T magnet will employ insulated ReBCO or NI-ReBCO; and whether to, and how to, incorporate an LTS outsert.

6. Complete the final system design.

Once items 1-6 above are complete, it is possible to commit to a cost and schedule for the first user magnet (goal of $25 \mathrm{~T}$ ). However, this first magnet will be a major step beyond the test coils and not all features will have been fully tested; some will be based on extrapolation. Therefore, this first $25 \mathrm{~T}$ user magnet needs to be developed through the proposed program on an R \& D contract, not a procurement contract. In addition, when items 1-6 are complete, we can define further development tasks required to enable a 30 T magnet to be designed and built and start those tasks in parallel with the construction of the 25 T magnet.

For this first magnet prototype-and all subsequent prototypes - to prove truly useful for the neutron and x-ray scattering communities, it must be designed as an integrated system for routine operation and frequent sample changes. The prototype must be optimized to minimize background contributions and must accommodate a wide range of sample environment systems. Unlike a conventional procurement where such requirements are listed in a specification, this magnet design will require significant and continuing collaboration with end users and commercial magnet manufacturers. The magnet development laboratory must leverage the experience of the commercial sector in building LTS magnets for x-ray and neutron scattering experiments, similar to what was done in the $32 \mathrm{~T}$ project. This experience includes split-coil designs and optics configurations that satisfy the continually developing requirements of a variety of experiments.

The magnet development laboratory needs strong and sustained interaction with user facilities as the design concept evolves, similar to what was accomplished during the HZB project. Only in this way, despite being the first magnets of their kind, will these prototype magnets be sufficiently robust for user operations. The $\mathrm{x}$-ray and neutron scattering communities have compared and contrasted requirements for a vertical-bore, split-coil magnet. Where possible, the requirements are made common in order to facilitate magnet development. Note that although x-ray and neutron user facilities are accustomed to dilution refrigeration inserts in magnets that can achieve temperatures down to around $50 \mathrm{mK}$, magnet development laboratories can leverage their significant experience to reach even lower temperatures (to around $10 \mathrm{mK}$ ), thereby extending phase space even further into the useful magnetic field/temperature $(\mathrm{B} / \mathrm{T})$ regime.

Additional capital investments at the initial stages of the project are key to enabling high field characterization and stress testing of conductors and components throughout the rest of this program. A large-bore superconducting magnet should be purchased to allow HTS coils and cables to be installed inside and tested to high-field, current-density, and mechanical strain as well as to perform fatigue testing. The magnet should be installed at the NHMFL, be part of the user facility, and be available to the community. A 15 T, $250 \mathrm{~mm}$ cold-bore magnet with a reverse-cryostat insert would be a good choice, but final parameters should be determined with input from the user community, which includes the fusion and high-energy physics communities, among others. A $20 \mathrm{kA}, 10 \mathrm{~V}$ DC power supply should be procured and used to test high-current cables installed in the large-bore magnet. 
The program will apply lessons learned from each prototype to inform the next and will increase field between prototypes at nominal 5 Tesla steps. An R \& D program must run in parallel to prepare for new conductor, coil, and integrated system technologies that can accommodate these increases in field and other improvements in magnet performance. The development budget and near-term goals need to evolve year by year based on how technology develops; as challenges are met for one magnet, the program must move on to what is required for the next. Note that $\mathrm{R} \& \mathrm{D}$ rarely happens on a predictable schedule. This recognition was important during the development of the $32 \mathrm{~T}$ magnet, as the NHMFL team learned from a number of test coils. Lessons from test coils will inform conductor suppliers of new requirements, spurring and directing additional conductor R \& D.

HTS material and coil technologies are improving rapidly, so while we know what development is required for the first magnet, for subsequent magnets we should be ready to take advantage of other emerging technologies. Today it is clear that fields higher than $17 \mathrm{~T}$ can be attained using insulated ReBCO and NI-ReBCO. However, just a few years ago, this was not so clear. In a couple of years' time it might be that $\mathrm{Bi}-2223, \mathrm{Bi}-2212$, or cabled conductors based on any one of these materials will be a better choice, partly due to progress from this program but also due to experience in the international high-field, high-energy, and fusion communities. Note that both SESP and MIT benefitted from changing HTS conductor emphasis from Bi-2223 to ReBCO, even though their applications were quite different.

Prior to committing to conductor technology for each subsequent magnet prototype, this program requires development tasks (similar to tasks 1-4 for the first magnet) to evaluate whichever conductor/coil technologies are most promising at the time. Also, one or more technologies may not demonstrate sufficient readiness at a given magnet decision point but might still hold sufficient promise to merit concerted R \& D through this program in order to improve readiness for the next magnet prototype at higher field. The priority for all these tasks, however, shall be lower than those tasks supporting the current magnet prototype development.

This R \& D program presents an opportunity for technology development in, and transfer to, the commercial sector. The LTS magnets, HTS materials, and, potentially, cables will require commercial development and supply. Costs of HTS magnets may come down low enough to enable a viable commercial market for condensed-matter physics magnets in user facilities, university labs, NMR, etc. Possibly even more important will be the enabling technologies for other applications.

If there are several labs that want copies of a particular successful prototype magnet, it should be possible to have copies made either on an R \& D contract or, possibly, a time and materials procurement contract, either from NHMFL or, eventually, from the commercial sector. Sample environment interfaces for toploading systems are well established and will only require careful specification of interfaces to enable most of the desired functionality.

\subsection{BEAMLINE PREPARATION}

While the prototype magnets are being built, the user facilities must prepare in parallel in order to be ready for these magnets. Design and construction of magnet prototypes is projected to take $3-5$ years each, while new $\mathrm{x}$-ray or neutron scattering instruments are projected to take $6-10$ years from concept to commissioning (longer if they are integrated with entirely new facilities). Sites at the various DOE-BES labs will need to be identified and work begun for beamlines to be ready for delivery of the magnet prototypes. Preparation will include providing additional support systems, mitigation of the impact of significant stray field on nearby components, preparation of personnel access zones, and study of stray field requirements for neighboring instruments. 
For at least the first magnet prototype at the SNS, an existing instrument or instruments (the Cold Neutron Chopper Spectrometer [CNCS] and the Hybrid Spectrometer [HYSPEC]) will be modified to accommodate the magnet. The SNS will generate a design study that will include recommended changes at these instrument locations, accounting for stray field and both space and utility requirements for additional support systems.

As subsequent magnets achieve higher and higher fields, it becomes less likely that either neutron or xray user facilities will have existing beamlines that can accommodate the magnets, even with modification. The first magnet may be built and ready before a dedicated beamline is built, but subsequent magnets with higher stray field will most likely need a specially prepared home. Therefore, new instruments designed around these new magnets need to be built in advance. Expected support systems also need to be identified, including helium recovery and recondensing systems that to date are not typically integral to either x-ray or neutron user facilities in the United States. Interfaces and overall dimensions must be defined to work both for the first and for subsequent magnets. Stray fields must be modeled for several optional coil technologies that could be used in the next prototype magnet. New instruments, in contrast to the magnet prototypes, are expected to be managed as capital projects, so we need a path forward to build a low-risk instrument that is planned to accommodate a moderate-risk prototype magnet.

\subsection{PROJECT MANAGEMENT}

A partnership between the public and private sectors as well as the user communities will be required for this proposal to be successful. To ensure project objectives and user needs are fully met, defined roles, responsibilities, authorities, and accountabilities (R2A2s) will be required. Defining the R2A2s will ensure each participant in the project clearly understands their role, the project objectives, and their deliverables and will reduce the potential for communication breakdowns. Depending on the entity, the R2A2s, objectives, and deliverables will be reflected in a variety of documents, including, but not limited to, memoranda of understanding (MOU), user agreements, Cooperative Research and Development Agreements (CRADA), material transfer agreements, Work for Others agreements (WFOs), and research and development subcontracts. Legally-binding agreements will address the management and disposition of any intellectual property that may be generated as part of this project. The agreements will also address proper attribution of the participants' (individuals, programs, and facilities) contributions to the project. In addition, nondisclosure agreements (NDAs) may be required for certain aspects of the project.

The success of the project will rely not only on clear R2A2s, but also on open, regular, and deliberate collaboration. Key contributors for a successful project must include experts in HTS magnet development, experts in HTS conductor manufacturing, LTS user magnet manufacturers catering to x-ray and neutron scattering, and the x-ray and neutron scattering community itself. In order to build upon prior deliverables and objectives at each step, the participants will hold quarterly meetings to transfer lessons learned to all participants. These quarterly meetings will be supplemented throughout the year with conference calls, video conferences, and webinars to ensure active collaboration and information exchange. In addition, the project will take advantage of available crowd sourcing and document repository technology, which will enable participants to connect on a daily basis.

The United States is already behind because the HZB magnet has been hosting users for over a year, and publications from those pioneering experiments are imminent. However, as described in this document, we see a clear path to a superior system by deploying the recent advances in HTS materials and coil technology. The workshop participants are excited to collaborate with the shared goal of advancing the frontier for UHF scattering experiments and realizing the great scientific potential of this new capability. 


\section{REFERENCES}

[1] Committee on Opportunities in High Magnetic Field Science, "Opportunities in High Magnetic Field Science," The National Academies Press, 2005.

[2] Committee to Assess the Current Status and Future Direction of High Magnetic Field Science in the United States, "High Magnetic Field Science and Its Application in the United States: Current Status and Future Directions," The National Academies Press, 2013.

[3] Basic Energy Sciences Advisory Committee, "Directing Matter and Energy: Five Challenges for Science and the Imagination," 2007.

[4] A. Wood, Magnetic Venture: the Story of Oxford Instruments, Oxford, UK: Oxford University Press, 2001.

[5] D. J. P. Morris et al., "Dirac Strings and Magnetic Monopoles in the Spin Ice Dy2Ti2O7," Science, vol. 326, p. 411-414, 2009.

[6] R. Cubitt et al., "Direct Observation of Magnetic Flux Lattice Melting and Decomposition in the High Tc Superconductor Bi2.15Sr1.95CaCu2O8+x," Nature, vol. 365, p. 407-411, 1993.

[7] A. D. Bianchi et al., "Superconducting vortices in CeCoIn5: Toward the Pauli-limiting field," Science, vol. 319, p. 177-180, 2008.

[8] M. B. Stone et al., "Extended Quantum Critical Phase in a Magnetized Spin-12 Antiferromagnetic Chain," Physical Review Letters, vol. 91, p. 037205, 2003.

[9] D. Tennant et al., "Unbound Spinons in the $S=1 / 2$ Antiferromagnetic Chain KCuF3," Physical Review Letters, vol. 70, no. 25, p. 4003-4006, 1993.

[10] R. Coldea et al., "Experimental Realization of a 2D Fractional Quantum Spin Liquid," Physical Review Letters, vol. 86, no. 7, p. 1335-1338, 2001.

[11] V. Kiryukhin et al., "Soliton Lattice in Pure and Diluted CuGeO3," Physical Review Letters, vol. 76, no. 24, p. 4608-4611, 1996.

[12] R. Coldea et al., "Direct Measurement of the Spin Hamiltonian and Observation of Condensation of Magnons in the 2D Frustrated Quantum Magnet Cs2CuCl4," Physical Review Letters, vol. 88, no. 13, p. 137203, 2002.

[13] K. Ross et al., "Quantum Excitations in Quantum Spin Ice," Physical Review X, vol. 1, no. 2, p. 021002, 2011.

[14] V. Kiryukhin et al., "Direct Observation of a Magnetic Field Induced CommensurateIncommensurate Transition in a Spin-Peierls System," Physical Review Letters, vol. 74, p. 1669, 1995.

[15] V. K. Pecharsky et al., "Giant Magnetocaloric Effect in Gd5(Si2Ge2)," Physical Review Letters, vol. 78, p. 4494, 1997.

[16] X. S. Xu et al., "Charge order, dynamics, and magnetostructural transition in multiferroic LuFe2O4," Physical Review Letters, vol. 101, p. 227602, 2008.

[17] L. Tan et al., "Spin-flop transition in Gd5Ge4 observed by x-ray resonant magnetic scattering and first-principles calculations of magnetic anisotropy," Physical Review B, vol. 77, p. 064425, 2008.

[18] Y. Feng et al., "Evolution of incommensurate spin order with magnetic field and temperature in the itinerant antiferromagnet GdSi," Physical Review B, vol. 88, p. 134404, 2013.

[19] J. Chang et al., "Direct observation of competition between superconductivity and charge density wave order in YBa2Cu3O6.67," Nature Physics, vol. 8, p. 871-876, 2012.

[20] A. T. Holmes et al., "A 17 T horizontal field cryomagnet with rapid sample change designed for beamline use," Review of Scientific Instruments, vol. 83, p. 023904, 2012. 
[21] P. Smeibidl et al., "First Hybrid Magnet for Neutron Scattering at Helmholtz-Zentrum Berlin," IEEE Transactions on Applied Superconductivity, vol. 26, no. 4, p. 4301606, 2016.

[22] O. Prokhneko et al., "Time-of-flight Extreme Environment Diffractometer at the HelmholtzZentrum Berlin," Review of Scientific Instruments, vol. 86, p. 033102, 2015.

[23] M. Bartkowiak et al., "The design of the inelastic neutron scattering mode for the Extreme Environment Diffractometer with the 26 T High Field Magnet," Nuclear Instruments and Methods in Physics Research A, vol. 797, p. 121-129, 2015.

[24] J. P. C. Ruff et al., "Susceptibility Anisotropy in an Iron Arsenide Superconductor Revealed by XRay Diffraction in Pulsed Magnetic Fields," Physical Review Letters, vol. 109, p. 027004, 2012.

[25] J. P. C. Ruff et al., "Magnetoelastics of a Spin Liquid: X-Ray diffraction Studies of Tb2Ti2O7 in Pulsed Magnetic Fields," Physical Review Letters, vol. 105, p. 077203, 2010.

[26] C. Detlefs et al., "Direct Observation of the High Magnetic Field Effect on the Jahn-Teller State in TbVO4," Physical Review Letters, vol. 100, p. 056405, 2008.

[27] Y. H. Matsuda et al., "High field x-ray diffraction study on a magnetic-field-induced valence transition in YbInCu4," Journal of the Physical Society of Japan, vol. 75, p. 024710, 2006.

[28] F. Duc et al., "Direct evidence of magnetostructural phase separation in the electron-doped manganite Ca0.8Sm0.16Nd0.04MnO3 by means of high magnetic field studies," Physical Review B, vol. 82, p. 054105, 2010.

[29] S. Gerber et al., "Three-Dimensional Charge Density Wave Order in YBa2Cu3O6.67 at High Magnetic Fields," Science, vol. 350, p. 6363, 2015.

[30] H. Nojiri et al., "Neutron Laue Diffraction Study of the Magnetic Phase Diagram of Multiferroic MnWO4 under Pulsed High Magnetic Fields," Physical Review Letters, vol. 106, p. 237202, 2011.

[31] K. Kuwahara et al., "Magnetic Structure of Phase II in U(Ru0.96Rh0.04)2Si2 Determined by Neutron Difraction under Pulsed High Magnetic Fields," Physical Review Letters, vol. 110, p. 216406, 2013.

[32] J. Beard, J. Billette, P. Frings et al, "Special Coils Development at the National High Magnetic Field Laboratory in Toulouse," Journal of Low Temperature Physics, vol. 170, no. 5, pp. 442-446, 2013.

[33] D. Haught et al., "Overview of the U.S. Department of Energy (DOE) High-Temperature Superconductivity Program for Large-Scale Applications," International Journal of Applied Ceramic Technology, vol. 4, no. 3, pp. 197-202, 2007.

[34] D. C. Larbalestier et al., "WTEC Panel Report on Power Applications of Superconductivity in Japan and Germany," 1997.

[35] D. Hazelton et al., "Recent Developments in 2G HTS Coil Technology," IEEE Transactions on Applied Superconductivity, vol. 19, p. 2218-2222, 2009.

[36] H. W. Weijers et al., "The generation of 25.05 T using a 2.11 T Bi2Sr2CaCu2Ox superconducting insert magnet," Superconducting Science and Technology, vol. 17, p. 636-644, 2004.

[37] K. Kinoshita, "HTS wire development and industrialization at Sumitomo," in 1st Workshop on Accelerator Magnets in HTS, Hamburg, Germany, 2014.

[38] Scheuerlien et al., "Void and phase evolution during the processing of Bi-2212 superconducting wires monitored by combined fast synchrotron micro-tomography and x-ray diffraction," Siuperconductivity Science and Technology, vol. 24, p. 115004, 2011.

[39] D. C. Larbalestier et al., "Isotropic round-wire multifilament cuprate superconductor for generation of magnetic fields above 30 T," Nature Materials, vol. 13, p. 375-381, 2014.

[40] H. W. Weijers et al., "Progress in the Development and Construction of a 32-T Superconducting Magnet," IEEE Transactions on Applied Superconductivity, vol. 26, no. 4, p. 4300807, 2016. 
[41] S. Hahn et al., "HTS Pancake Coil Without Turn-to-Turn Insulation," IEEE Transactions on Applied Superconductivity, vol. 21, p. 1592-1595, 2011.

[42] A Godeke et al., "A feasibility study of high-strength Bi-2223 conductor for high-field solenoids," Superconductor Science and Technology, 2017 (pending).

[43] W. H. Fietz et al., "High-Current HTS Cables: Status and Actual Development," IEEE Transactions on Applied Superconductivity, vol. 26, p. 4800705, 2016.

[44] T. Shen et al., 5-10 kA class Bi02212 Rutherford cables carrying Je of $500 \mathrm{~A} / \mathrm{mm} 2$ at $4.2 \mathrm{~K}$ and 20 T, Denver, CO: talk at Applied Superconductivity Conference, 2016.

[45] N. M. Strickland et al., "A 1 kA-class cryogen-free critical current characterization system for superconducting coated conductors," Review of Scientific Instruments, vol. 85, p. 113907, 2014.

[46] Z. Islam et al., "Proposal, High Magnetic Field Sector for X-Ray Scattering," 2016.

[47] APR-U Beamline Review Committee, "High Magnetic Field Sector for X-Ray Scattering (HFM)," 2016.

[48] P. D. Noyes et al., "Protection heater development for REBCO coils," IEEE Transactions on Applied Superconductivity, vol. 22, p. 4704204, 2014.

[49] S. Hahn et al., "Operation and performance analyses of 350 and $700 \mathrm{MHz}$ low-/high-temperature superconductor nuclear magnetic resonance magnets: A march toward operating frequencies above 1 GHz," Journal of Applied Physics, vol. 105, p. 024501, 2009.

[50] S. Yoon et al., "26 T 35 mm all-GdBa2Cu3O7-x multi-width no-insulation superconducting magnet," Superconductivity Science and Technology, vol. 29, p. 04LT04, 2016.

[51] J. Song et al., "Over-current quench test and self-protecting behavior of a $7 \mathrm{~T} / 78 \mathrm{~mm}$ multi-width no-insulation REBCO magnet at 4.2 K," Superconductivity Science and Technology, vol. 28, p. 114001, 2015.

[52] S. Hahn et al., "No-insulation multi-width winding technique for high temperature superconducting magnet," Applied Physics Letters, vol. 103, p. 173511, 2013.

[53] Z. Melhem et al., "Development of Bi-2212 Insert Coils for Ultra High Field Magnet Applications," Physics Procedia, vol. 36, p. 805-811, 2012.

[54] W. D. Markiewicz et al., "Design of a superconducting 32 T magnet with REBCO high field coils," IEEE Transactions on Applied Superconductivity, vol. 22, p. 4300704, 2012.

[55] J. Bascuñán et al., "90-mm/18.8-T all-HTS insert magnet for 1.3 GHz LTS/HTS NMR application: magnet design and double pancake coil fabrication," IEEE Transactions on Applied Superconductivity, vol. 24, p. 4300904, 2014.

[56] S. Yoon et al., "26T 35mm all-GdBaCuO multi-width no-insulation superconducting magnet," Superconductivity Science and Technology, vol. 29, p. 04LT04, 2016.

[57] S. Hahn, Not yet published; results presented in ASC2016 conference, 2016.

[58] A. Godeke et al., "Development of wind-and-react Bi2212 accelerator magnet technology," IEEE Transactions on Applied Superconductivity, vol. 18, p. 516-519, 2008.

[59] C. Barth et al., "Temperature- and field-dependent characterization of a conductor on round core cable," Superconducting Science and Technology, vol. 8, p. 065007, 2015.

[60] M. Takayasu et al., "Present status and recent developments of the twisted stacked-table cable conductor," IEEE Transactions on Applied Superconductivity, vol. 26, p. 6400210, 2016.

[61] J. Fleiter et al., "Characterization of Roebel Cables for Potential Use in High-Field Magnets," IEEE Transactions on Applied Superconductivity, vol. 25, 2015.

[62] D. C. van der Laan et al., "Record current density of $344 \mathrm{~A} / \mathrm{mm} 2$ at $4.2 \mathrm{~K}$ and $17 \mathrm{~T}$ in CORC® accelerator magnet cables," Superconductivity Science and Technology, vol. 29, p. 055009, 2016. 
[63] J. D. Weiss et al., "Introduction of CORC® wires: highly flexible, round high-temperature superconducting wires for magnet and power transmission applications," accepted, Superconductor Science and Technology, 2016. 


\section{APPENDIX A. CANDIDATE TECHNOLOGIES FOR UHF MAGNETS}

\section{A.1 INSULATED REBCO TECHNOLOGY FOR NHMFL’S 32 T MAGNET}

Owing to the successful demonstration of the $7 \mathrm{~T}$ coil in a $20 \mathrm{~T}$ background field, NHMFL initiated its

own program for testing a few small coils with SuperPower tape and submitted a proposal to NSF to build a 32 T HTS/LTS user magnet for condensed matter physics experiments. A grant was awarded in 2010, and the resulting magnet [40] employs an HTS insert that utilizes ReBCO tape from SuperPower and an LTS outsert that utilizes a new class of high-field, wide-bore LTS magnets. This outsert features highperformance $\mathrm{Nb}_{3} \mathrm{Sn}$ wire manufactured by OST using restacked rod process (RRP) wire. This magnet has been fully assembled and is presently in the testing and commissioning phase.

The NHMFL had been working on HTS magnets and materials since 1991. However, when it started working with SuperPower's ReBCO tape in 2007, a tremendous amount of technology still needed to be developed. The NHMFL team built several test coils, and in each test unknown-unknowns were uncovered. NHMFL also conducted extensive fatigue testing of materials and components [33]. As a result of all this experimentation and testing, NHMFL developed key technologies and procedures required for a reliable user magnet including: (1) characterizing the ReBCO tape and working with the supplier to improve its quality, (2) developing ultra-thin insulation to allow operation at high $J_{e}$ and stress, (3) developing a methodology to wind pancake coils using non-uniform tape, and (4) developing highstrength joints that could operate in high field.

A particularly difficult challenge overcome by new technology was quench protection. Over the years, many LTS magnets have been damaged or destroyed through quenches - the rapid transition from superconducting to normal state, which converts the energy stored in the inductance of the coil into heat in the magnet. The LTS magnet community has developed the means to protect these systems, which frequently requires distributing the energy uniformly over the coils. Protecting HTS coils is much more difficult than protecting LTS coils because the high critical temperature results in the normal zone growing very slowly, resulting in excessive localized heating that causes the conductor to experience excessive thermal strain, melting, or even burning. The NHMFL developed a quench detection and protection system based on steel heaters stacked within the coil pack. When a quench is detected, batteries send $1 \mathrm{kA}$ of current into the heater circuit to heat the conductor every few centimeters. Over 100 intentional quenches have been generated in the ReBCO coils without performance degradation [48].

\section{A.2 NO-INSULATION REBCO TECHNOLOGY}

In 1999, a group led by Y. Iwasa at MIT’s Francis Bitter Magnet Laboratory submitted a 3-phase proposal to the National Institutes of Health to build a 1.0 GHz (23 T) NMR magnet that consists of an HTS insert and an LTS outsert. Initially, the team proposed to use Bi-2223 tape for the HTS insert, but later in 2009-after significant technical progress in ReBCO conductor technology — they decided to upgrade the final phase goal to $1.3 \mathrm{GHz}(30.5 \mathrm{~T}$ ) by using SuperPower's ReBCO tape for a $800 \mathrm{MHz}$ $(18.8 \mathrm{~T})$ insert [49]. To attain the $30 \%$ higher field while staying within the constraints for mechanical stress, protection, schedule, and funding, this group developed the no-insulation ReBCO technology (NIReBCO). In this approach, and as the name suggests, the conductor is wound into a coil without electrical insulation.

In traditionally-insulated superconducting magnets (e.g., most LTS and HTS magnets, including the $32 \mathrm{~T}$ magnet at NHMFL), when a quench occurs, current is forced to flow through the normal (quenched) zone, which produces intense heating. By foregoing the insulation, the current is no longer constrained to flow through this normal zone at high current density. Instead, current can now flow into neighboring 
layers, bypassing the normal zone, which results in much less intense heating -making the NI-ReBCO coil effectively self-protecting when a local quench occurs [41]. Worldwide, several groups are now publishing papers on this approach and its variations. To date, more than 10 multi-pancake no-insulation (NI) test coils have been designed and built, and their self-protecting feature has been successfully demonstrated [50], [51], [52].

While it is possible to make a 30-T solenoid entirely from HTS materials (no LTS), this is atypical due to cost and risk. Instead, two different approaches have been taken to produce high-field magnets with an HTS insert and LTS outsert. The first school of thought is that the HTS component is high risk, so its size should be minimized and as much field as possible should be provided by the LTS part of the magnet. This approach was seen in the early work of a number of organizations where an LTS section provided around $20 \mathrm{~T}$ [53]. The second school says that to minimize cost one needs to minimize stored energy or mass. This approach has been taken more recently in NHMFL's 32 T magnet where the LTS section only provides 15 T [54] and also in MIT's proposed 30 T NMR magnet where the LTS section would provide only $11.7 \mathrm{~T}$ [55].

That said, the NI-ReBCO approach offers a third option: no LTS section at all. By leaving out the insulation, one can reduce the amount of copper and attain extremely high current densities. By not using an LTS section, the overall magnet can become smaller than would be possible in an LTS/HTS approach and quench protection becomes simpler. This approach was taken in a $26 \mathrm{~T}$, all-HTS magnet developed by S. Hahn et al. and tested at NHMFL in 2015. This $26 \mathrm{~T}$ magnet has an outer diameter of only $172 \mathrm{~mm}$ [56]. While this approach clearly produces the most compact design, and therefore the lowest stray field and instrument footprint, it is not yet clear which approach results in lowest cost (HTS materials are typically quite expensive). Furthermore, in preliminary tests NI coils also prove robust-even in the presence of deliberately-made imperfections in the HTS tape. This imperfection insensitivity implies substantial enhancement in operational reliability and may result in relaxed specifications to the tape manufacturers, which might result both in longer lengths for the tape pieces and in lower cost.

One potential limitation of NI for application in x-ray and neutron scattering experiments is the relatively long time expected for ramping and stabilization when compared to insulated ReBCO designs under similar conditions. However, there is active R \& D underway to reduce ramping and stabilization time for $\mathrm{NI}$; these development efforts are pursuing several strategies, including partial insulation, metallic insulation, and active feedback control. Recent experiments with a new NI coil demonstrated around 20 times faster charging without sacrificing the self-protection feature [57]. This new NI test coil is a multipancake, no-split configuration with an inner diameter of $100 \mathrm{~mm}$, a field of $3 \mathrm{~T}$ in the middle of the coil at $200 \mathrm{~A}$, and exhibits a barely-discernible charging delay due to the short charging time constant facilitated by the new approach. Demonstration of this faster ramping in a full-scale magnet is planned at NHMFL. Thus, NI-ReBCO is now showing promise to enable very compact, lower stray field, and less expensive magnets in the 25-30 T range than is possible with insulated ReBCO.

\section{A.3 MULTI-STRAND HTS CABLE MAGNETS}

A few organizations are now developing multi-strand cables based on HTS conductors. Although these cables are being developed for use in large non-solenoid magnetic field generating systems such as highenergy physics dipoles and fusion coils, they may provide an alternative to high-field solenoid magnets wound from single-tape or single-strand, with performance that may be better optimized for some experiments. To date, most of the cable development work has been based on ReBCO tape but there is also some work based on Bi-2212 strand [58], [59], [60], [61], [43].

High-field magnets wound from ReBCO cables have a promising but untested advantage over single-tape magnets. The cable would provide some redundancy if HTS strand or tape quality is too poor to allow 
single-strand construction. Current sharing between tapes in the cable (similar to NI technology) would allow current to bypass these dropouts, thereby reducing the requirements and thus cost of the ReBCO tapes in the cable. The approximately 10 times higher current handling capacity of cables enables fewer turns for the same field at sample, lowering inductance. The much lower inductance, and for some cables their tape twisting strategies, enable very high ramp rates, offer the potential of much easier quench protection, and potentially result in lower levels of magnet heating during ramping. Advanced Conductor Technologies, LLC (ACT) pioneered the conductor on round core $\left(\mathrm{CORC}^{\circledR}\right)$ cable, which features the smallest bending radii and is therefore the most suitable cable for small-bore UHF solenoids. CORC cables contain a high number of ReBCO tapes that are wound with a small pitch around a thin former, making them highly flexible and allowing them to have a very high $J_{e}$ at high field [62]. CORC cables have already demonstrated an $J_{e}$ of $344 \mathrm{~A} / \mathrm{mm}^{2}$ at $17 \mathrm{~T}$, although that current density is limited in part by the tape surface being oriented normal to field direction, twice per twist pitch, around the cable former. ACT recently demonstrated CORC coils with bores as small as $50 \mathrm{~mm}$ in diameter [63], and is planning to test a 3-5 T insert magnet with a bore of only $60 \mathrm{~mm}$ in a $14 \mathrm{~T}$ background field at NHMFL in 2017.

In addition to ReBCO cabling, efforts to develop Rutherford cabling made of Bi-2212 are also underway. Rutherford Bi-2212 cabling allows for a high packing factor (>90 \%), and new pancake designs employing this cabling facilitate precise temperature control of the Bi-2212 coils in a small furnace volume, thereby addressing the challenges associated with the reaction of $\mathrm{Bi}-2212$ coils. The successful reaction of racetrack coils (double pancake like coils) was recently demonstrated by a collaboration between Lawrence Berkeley National Lab (LBNL) and NHMFL [44]. The cables feature low inductance, easy quench protection, robustness, and fast ramping, and might enable cooling of magnets via a cryocooler system instead of liquid helium.

Despite all of the developments and potential advantages outlined in this section, cables have not yet matched the $J_{e}$ of single-strand construction, leading to larger coil diameters, larger stray fields, and most likely lower maximum achievable field at sample compared to single strand geometries. Moreover, this program would not supplant cable development tasks supported through other programs like Bi-2212 reinforcement. However, we may find cable coils to be an attractive option for future prototype magnets, and so our research will benefit from targeted coil tests using these cables, which may in turn inform subsequent cable refinement. 